\title{
Robotic Assist for MR-Guided Surgery Using Leverage and Parallelepiped Mechanism
}

\author{
Yoshihiko Koseki ${ }^{1}$, Kiyoyuki Chinzei ${ }^{1}$, Noriho Koyachi ${ }^{1}$, and Tatsuo $\mathrm{Arai}^{2}$ \\ 1 Mechanical Engineering Laboratory, AIST, MITI, \\ 1-2 Namiki, Tsukuba, Ibaraki 305-8564, Japan \\ \{koseki, chin, koyachi\}@mel.go.jp \\ http://www.mel.go.jp/ \\ 2 Graduate School of Engineering Science, Osaka University, \\ 1-3 Machikaneyama-cho, Toyonaka, Osaka 560-8531, Japan \\ arai@sys.es.osaka-u.ac.jp \\ http://www-arailab.sys.es.osaka-u.ac.jp/
}

\begin{abstract}
In this paper, we would propose a novel mechanism of surgical manipulator, which assists the surgeon in precise positioning and handling of surgical devices, like biopsy needle, endscope, in MR-guided surgery. This mechanism can transmit 3 translational and 3 rotational motion from the outside to the inside of MR imaging area using leverage and parallelepiped mechanism. Such a remote actuation is significantly helpful for robotic assist under MR-guided surgery because the strong magnet of MR denies the existence of magnetic and electric devices around imaging area. This mechanism also has merits of the mechanical safety and simple shape. The combination of stereotactic imaging and precise positioning would enable a less invasive surgery in brain and spine surgery.
\end{abstract}

\section{Introduction}

\subsection{Robotic Assist for Image-Guided Surgery}

The image-guided surgery is a technique to reach a surgical instrument to a target through a narrow access under observation of X-ray CT and to minimize the damage to normal tissue. The trajectory to the target is strategically decided and the treatments must be executed precisely according to the pre-operative planning. So the precise positioning is one of the keys of this operation.

A stereotactic neurosurgery is the most typical example because it has urgent requirement to avoid surgical trauma. Many researchers have proposed robotic assist [1], [2]. Although it is much difficult for a surgeon to aim manually at concealed target inside skull, a robot potentially provides precise positioning because of its numerical control referring to the coordinate frame of X-ray CT image. Not only less invasion but also robotic surgery is expected to be effective on avoiding surgeon's infection and reducing the number of surgeons.

On the other hand, the advantage of MRI in comprehending the pathobiology of soft tissue leads to an image-guided surgery under MRI, especially in brain and 
spine surgery [3]. However, many conventional devices and instruments cannot be used because they affect the magnetic field and causes serious noise and deformation of images. Furthermore, the magnet surrounds the patient so closely that the accessibility of surgeon is also restricted. In these days, surgeons are capable of accessing to patient due to development of interventional MRI, socalled open MRI.

Some researchers have studied robotic assist under MRI for the same motivation as that under X-ray CT [4]. This MR manipulator has much more technical difficulties because the conventional mechatronical components are not MR compatible. So MR-guided robotic surgery needs comprehensive research of image-navigation, MR compatible mechatronics, and surgical manipulator.

\subsection{MR Compatible Manipulator Project}

Our group started open MR compatible manipulator project at 1996 and targets to develop a surgical manipulator, which works around open MRI of GE's Signa $\mathrm{SP}^{\mathrm{TM}}$, so-called double doughnut type. We have been studying the following three topics.

The first is navigation and registration of coordinate by MRI. Hata reports the navigation method under MR [5].

The second is, the key study of this project, MR compatibility of material, shape, arrangement, and electrical design of mechatronical components. Chinzei reported the design criteria [6], defining MR compatibility and discussing mechatronical components systematically. And we temporally conclude that remote actuation results better MR compatibility.

This paper would study the third topic, mechanism design of the MR compatible manipulator. This topic has common problems to all surgical manipulators, like mechanical safety, interface safety, sterilization compatibility. Many researchers have proposed unique mechanisms for surgical manipulators [4], [7]. The MR compatible manipulator must satisfy both MR compatibility and these requirements.

To solve these problems, we would propose a novel mechanism of surgical manipulator introduced in following sections.

\section{Leverage and Parallelepiped Mechanism}

\subsection{Mechanism}

Our novel mechanism, LPM (Leverage and Parallelepiped mechanism) can transmit full set of spatial motion, 3 translational and 3 rotational motion. Its input is attached to a conventional manipulator and its output is allocated into the workspace around MR gantry.

Fig. 1 schematically explains the structure and functions of LPM. The lever is supported by a gimbal via rotating and sliding pair. The gimbal is fixed on the base and works as a fulcrum of the lever. 


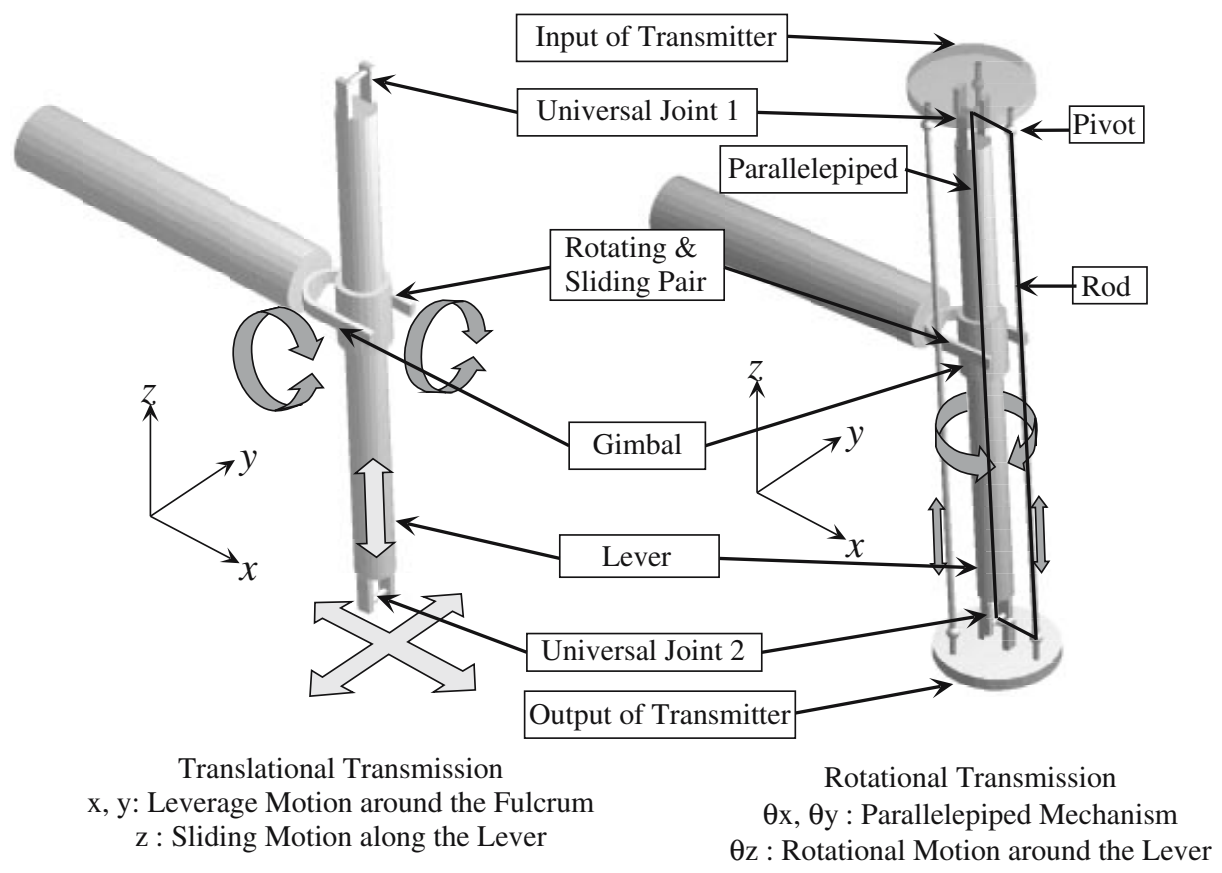

Fig. 1. Leverage and Parallelepiped Mechanism

As shown in the left of Fig. 1, the swinging motion around the fulcrum transmits the translational motion in $\mathrm{x}$ - and y-axes. The sliding motion along the lever allowed by the rotating and sliding pair transmits the translational motion in z-axis.

As shown in the right of Fig. 1, the rotating and sliding pair allows the rotational motion around z-axis. The input and output plates are connected with the lever via universal joint 1 and 2 , respectively. The universal joints transmit the rotational motion around $\mathrm{z}$-axis but never constrain the rotational motion around $\mathrm{x}$ - and $\mathrm{y}$-axes.

Some rods joint the input and output plates via pivots. The rods are as long as the lever and are connected to the plates at relatively same positions. So the pairs of rod and lever form parallelepipeds. These parallelepiped mechanisms always keep the input and output plates in parallel and transmit rotational motion around $\mathrm{x}$ - and $\mathrm{y}$-axes. If the rods can endure both expansion and contraction, 2 rods are necessary for 2 rotational motion and sufficient unless the lever and rods are in same plane. If the rods can endure only expansion, 3 rods are necessary and sufficient unless any 3 of the rods and the lever are in same plane. The latter case is more practical because a long rod cannot endure high pressure. If wires 
are used for the rods, the pivots are not necessary. Actually, our prototype has 4 wires in order to avoid confliction. (See Fig. 2(b).)

In Fig. 1, the rods are outside of the lever. In this case, the rotation range around z-axis is limited by the conflict between rods and gimbal. Therefore, our prototype has rods inside the lever as shown in Fig. 2(b).

\section{$2.2 \quad$ Features}

Remote Actuation Any electromagnetic actuator and a magnetic metal must not enter the neighborhood MR gantry. As for a fluid motor, actuation is free from magnet and electricity but its control system consists of magnetic valves. Even An ultrasonic motor, which doesn't include any magnetic body, makes noise to MR image, while working if it's close to MR imaging area. So the remote actuation is currently the best solution for MR manipulator, and LPM is one of the remote actuation mechanisms.

Mechanical Safety A serial link manipulator, which is widely used in factories, has potential danger of conflict between elbow joint and surgeon. Because an articular manipulator causes the motion of elbow, which is difficult for surgeon to forecast. LPM doesn't have such danger, because it's always straight. The wider workspace expands the potentiality of danger because everything in the workspace might be damaged when the manipulator runs out of control. As for LPM, the output workspace can be restrained by the input workspace. For example, if a barrier is set to the left in input workspace, the end-effector in output workspace cannot move to the right. Accordingly, LPM has potentiality of workspace control by double workspace, but this workspace control needs further study.

Simple Shape Even in robotic assist surgery, the operation is performed mainly by surgeon and robot's motion must be supervised by surgeon. Therefore, there should be left a space for surgeon(s). Because LPM has thin and straight shape and actuators and controllers are distant from the workspace, it doesn't occupy large space around the patient.

Demerits Because LPM is attached to a manipulator in serial, the backlash and deformation under load of LPM are added to that of the manipulator. So LPM potentially decreases the stiffness. As for the translational deformation and backlash except along the lever, the deformation and backlash originated in the manipulator affect to the output in proportion to the ratio of the output lever length to input lever length (See. Eq. (1), (11)). So, elasticity of LPM should be analyzed at the time of design.

The inclination of the output is limited by the limit angle of pivot, universal joints, and by conflict between the lever and rods. The translational displacement along the lever is restricted by the sliding range of sliding and rotating pair. The translational workspace changes according to the ratio of the output lever length to input lever length. So, allowable input must be analyzed at the time of design. 


\subsection{Current Prototype}

We made a prototype of LPM manipulator for so-called double doughnut type of open MRI (See Fig. 2(a)). The purpose of this prototype is to make clear mechanical problems. So this prototype is not MR compatible but all components are designed to be exchangeable with MR compatible components. The reasons are that MR compatibility of mechatronics is studied in another paper (second topic), and that MR compatible components are very expensive. For example, titanium has good MR compatibility and biocompatibility but costs very much. For the same reason, the back drivability of manipulator is not implemented to the current prototype.

The manipulator, which drives the input of LPM, is decided to fixed-linear parallel mechanism (FLP) [8]. The FLP is one of parallel mechanisms, has 6 linear actuators fixed on the base, and has 6 middle-links, which connect between the endplate and actuators in parallel (See Fig. 2(a)). FLP has good property of remote actuation because its actuators always stay in the same positions distant from MR gantry. FLP has demerit that it has narrow workspace especially in orientation but parallelepiped mechanism also has narrow workspace in orientation. So this demerit is not bottleneck of workspace. Those are why FLP is used.

Kinematic parameters are decided by conventional Monte-Carlo method to meet the workspace of $\pm 100[\mathrm{~mm}]$ in $\mathrm{x}-, \mathrm{y}^{-}, \mathrm{z}$-axes, and \pm 30 [deg.] around $\mathrm{x}-, \mathrm{y}-$, z-axes.

\section{$3 \quad$ Kinematics and Statics}

In this section, some important kinematic and static equations of LPM are obtained. All notations are illustrated and noted in Fig. 3. The origins and power points of input and output are assumed to be located at the crossing points of universal joints, respectively.

Firstly, the forward kinematics is shown in the followings. The position and orientation of LPM's output are obtained. The inverse kinematics is omitted here.

$$
\begin{aligned}
& \boldsymbol{p}_{\text {output }}=-l_{\text {lever }} \boldsymbol{q}+\boldsymbol{p}_{\text {input }} \\
& \boldsymbol{R}_{\text {output }}=\boldsymbol{R}_{\text {input }}
\end{aligned}
$$

Secondly, the forward statics and the force exerted on the fulcrum are obtained.

$$
\begin{gathered}
\boldsymbol{f}_{\text {output }}=-\frac{\left|\boldsymbol{p}_{\text {input }}-\boldsymbol{p}_{\text {fulcrum }}\right|}{\left|\boldsymbol{p}_{\text {output }}-\boldsymbol{p}_{\text {fulcrum }}\right|} \boldsymbol{f}_{\text {input }} \\
+\frac{l_{\text {lever }}}{\left|\boldsymbol{p}_{\text {input }}-\boldsymbol{p}_{\text {fulcrum }}\right|}\left(\boldsymbol{q} \cdot \boldsymbol{f}_{\text {input }}\right) \boldsymbol{q}
\end{gathered}
$$


Note that $\boldsymbol{a} \cdot \boldsymbol{b}$ is inner product of $\boldsymbol{a}$ and $\boldsymbol{b}$.

$$
\begin{aligned}
\boldsymbol{m}_{\text {output }} & =\boldsymbol{m}_{\text {input }} \\
\boldsymbol{f}_{\text {fulcrum }} & =\boldsymbol{f}_{\text {input }}-\boldsymbol{f}_{\text {output }}
\end{aligned}
$$

Thirdly, infinitesimal displacements of output are obtained. They are corresponding to velocity and elastic deformation.

$$
\begin{gathered}
\delta \boldsymbol{p}_{\text {output }}=-\frac{\left|\boldsymbol{p}_{\text {output }}-\boldsymbol{p}_{\text {fulcrum }}\right|}{\left|\boldsymbol{p}_{\text {input }}-\boldsymbol{p}_{\text {fulcrum }}\right|} \delta \boldsymbol{p}_{\text {input }} \\
+\frac{l_{\text {lever }}}{\left|\boldsymbol{p}_{\text {input }}-\boldsymbol{p}_{\text {fulcrum }}\right|}\left(\boldsymbol{q} \cdot \delta \boldsymbol{p}_{\text {input }}\right) \boldsymbol{q} \\
\delta \boldsymbol{R}_{\text {input }}=\delta \boldsymbol{R}_{\text {output }}
\end{gathered}
$$

Here, it should be noticed that the elasticity of LPM is proportional to the square of the ratio of the output lever length to input lever length. If the output lever is 2 times as long as input lever, the stiffness of LPM is $1 / 4$ of that of input manipulator.

$$
\begin{gathered}
\boldsymbol{f}_{\text {input }}=-\frac{\left|\boldsymbol{p}_{\text {output }}-\boldsymbol{p}_{\text {fulcrum }}\right|}{\left|\boldsymbol{p}_{\text {input }}-\boldsymbol{p}_{\text {fulcrum }}\right|} \boldsymbol{f}_{\text {output }} \\
\delta \boldsymbol{p}_{\text {input }}= \\
\text { The first term of Eq. (3) }
\end{gathered}
$$

$\boldsymbol{K}$ is Flexibility Matrix of the input manipulator

$$
\begin{aligned}
\delta \boldsymbol{p}_{\text {output }}= & -\frac{\left|\boldsymbol{p}_{\text {output }}-\boldsymbol{p}_{\text {fulcrum }}\right|}{\left|\boldsymbol{p}_{\text {input }}-\boldsymbol{p}_{\text {fulcrum }}\right|} \delta \boldsymbol{p}_{\text {input }} \\
\text { The first term of Eq. (6) } & \\
= & \left(\frac{\left|\boldsymbol{p}_{\text {output }}-\boldsymbol{p}_{\text {fulcrum }}\right|}{\left|\boldsymbol{p}_{\text {input }}-\boldsymbol{p}_{\text {fulcrum }}\right|}\right)^{2} \boldsymbol{K} \boldsymbol{f}_{\text {output }}
\end{aligned}
$$

The following equations describe the connection between the input manipulator and input of LPM.

$$
\begin{array}{rlrl}
\boldsymbol{p}_{\text {input }} & = & \boldsymbol{p}_{\text {endplate }}+\boldsymbol{R}_{\text {endplate }} \boldsymbol{p}_{\text {etoi }} \\
\boldsymbol{R}_{\text {input }} & = & \boldsymbol{R}_{\text {etoi }} \boldsymbol{R}_{\text {endplate }} \\
\boldsymbol{f}_{\text {input }} & = & \boldsymbol{f}_{\text {endplate }} \\
\boldsymbol{m}_{\text {input }} & =\boldsymbol{m}_{\text {endplate }}+\boldsymbol{p}_{\text {etoi }} \times \boldsymbol{f}_{\text {endplate }}
\end{array}
$$

Note that $\boldsymbol{a} \times \boldsymbol{b}$ is outer product of $\boldsymbol{a}$ and $\boldsymbol{b}$. 
The kinematics of FLP is shortly introduced here but the details are shown in [8]. The inverse kinematics can be obtained by solving the following equations individually for $l_{\text {linear }_{i}}$. The forward kinematics can be obtained by solving the following equations simultaneously for $\boldsymbol{p}_{\text {endplate }}$ and $\boldsymbol{R}_{\text {endplate }}$.

$$
\begin{array}{r}
\left|\left(\boldsymbol{p}_{\text {endplate }}+\boldsymbol{R}_{\text {endplate }} \boldsymbol{p}_{\text {etol }_{i}}\right)-\left(l_{\text {linear }_{i}} \boldsymbol{v}_{\text {linear }_{i}}+\boldsymbol{p}_{\text {btol }_{i}}+\boldsymbol{p}_{\text {base }}\right)\right|=l_{\text {link }_{i}}( \\
\text { while } i=1 \cdots 6
\end{array}
$$

\section{Conclusions and Future Works}

In this paper, we proposed our novel and unique mechanism of surgical manipulator, leverage and parallelepiped mechanism. Its possible application is precise positioning and handling of biopsy needle, endscope and other surgical devices under MR-guided surgery. We introduced the structure and functions, discussed the merits and demerits and formulated the kinematics and statics.

The current prototype is shortly introduced but it's still under development. We are planning to test its basic experiment like precision and stiffness, and preclinical evaluations.

\section{References}

1. Burckhardt C.W., Flury P., Glauser D.: Stereotactic Brain Surgery, IEEE Engineering in Medicine and Biology Magazine, Vol. 14, No. 3, pp. 314-317, May/June 1995

2. Kwoh Y.S., Hou J., Jonckheere E., Hayati S.: A Robot with Improved Absolute Positioning Accuracy for CT Guided Stereotactic Brain Surgery, IEEE tran. on Biomedical Engineering, Vol. 35, No. 2, pp. 153-160, Feb. 1988

3. Jolesz F.A., Blumenfeld S.G.: Interventional Use of Magnetic Resonance Imaging, Magn Reson Q, 1994, Vol. 10, No. 2, pp. 85-96

4. Masamune K., Kobayashi E., et al.: Development of a MRI Compatible Needle Insertion Manipulator for Stereotactic Neurosurgery, Proc. of MRCAS'95, Baltimore MD, pp. 165-172, Nov. 1995

5. Hata et al.: Multimodality Deformable Registration of Pre- and Intraoperative Images for MRI-Guided Brain Surgery, Proc. of MICCAI'98, pp. 1067-1074, 1998

6. Chinzei K., Kikinis R., Jolesz F.: MR Compatibility of Mechatronic Devices: Design Criteria, Proc. of MICCAI'99, pp. 1020-1031, 1999

7. Taylor R., et al.: A Telerobotic Assistant for Laparoscopic Surgery, IEEE Engineering in Medicine and Biology Magazine, Vol. 14, No. 3, pp. 279-288, May/June 1995

8. Arai T., Tanikawa T., Merlet J.P., Sendai T.: Development of a New Parallel Manipulator with Fixed Linear Actuator, Proc. of Japan/USA Symposium on Flexible Automation, pp. 145-149, 1995 


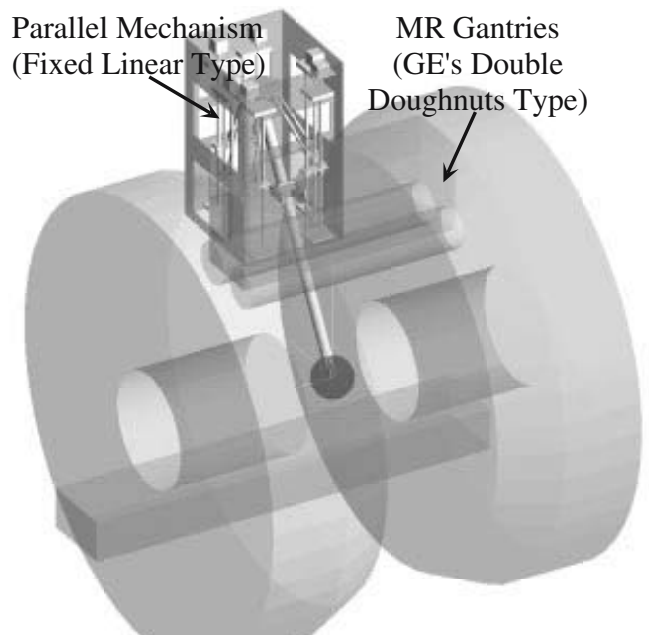

(a) CG View of Prototype and Open MRI

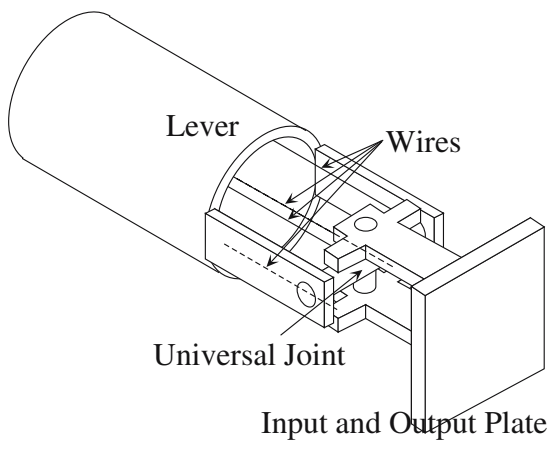

(b) End of Lever and Parallelepiped Mechanism of Prototype
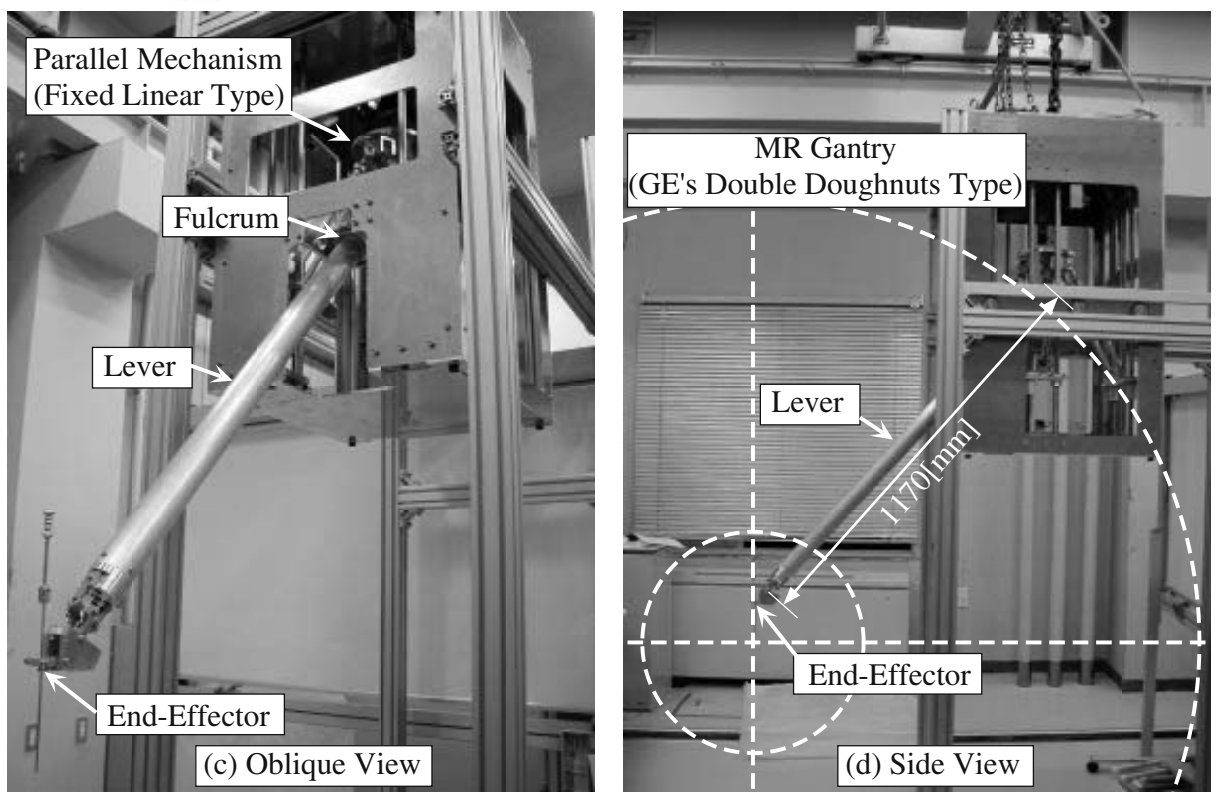

Fig. 2. Current Prototype 


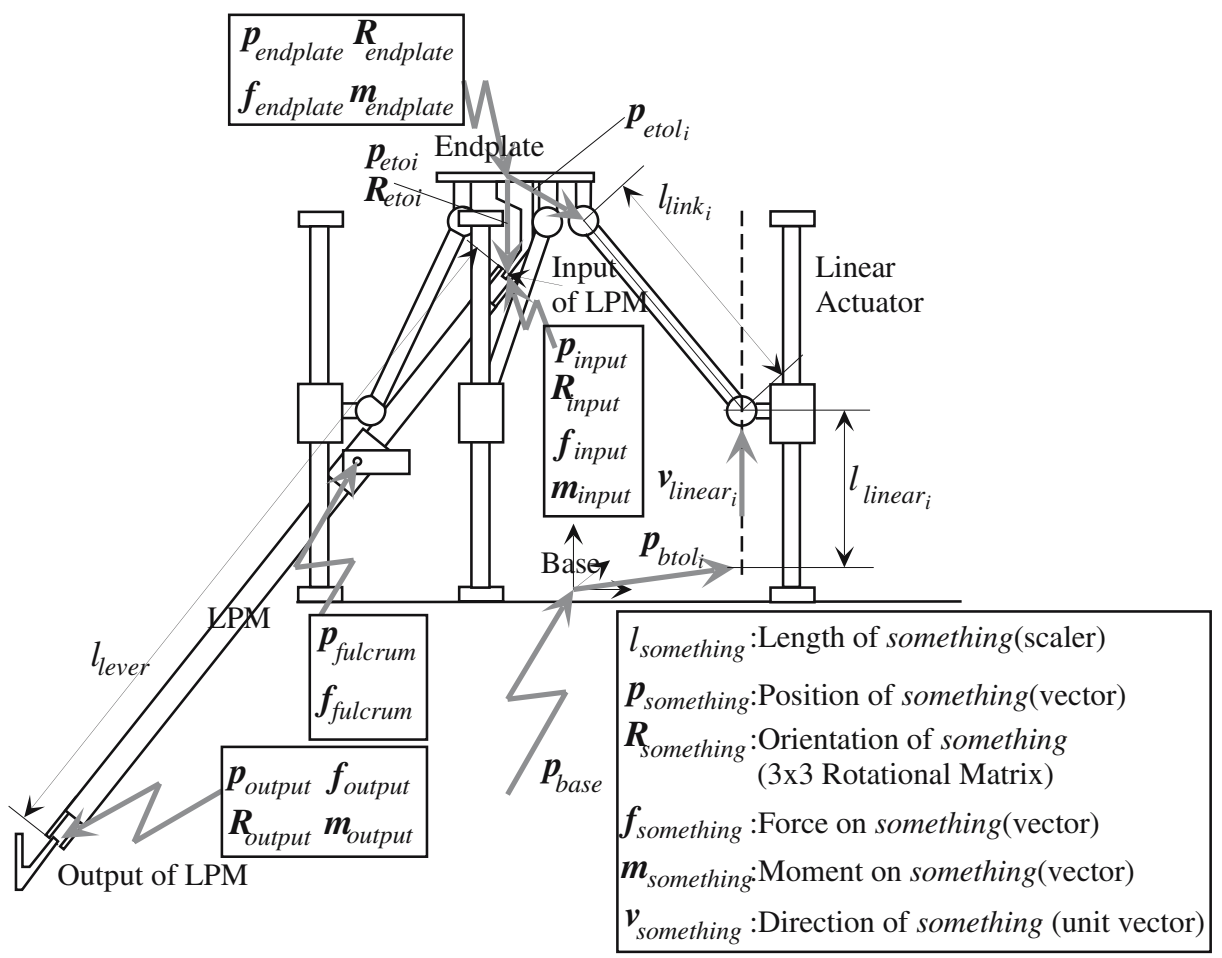

Fig. 3. Notataions in Equations 